\title{
Mitigating the impact of SARS-CoV-2 on residential facilities for persons with intellectual disability and/or autism spectrum disorder: two experiences from the Italian red zone
}

\author{
Jacopo Santambrogio, Michela Russo, Sergio Terrevazzi, Gianluca Peschi, \\ Massimo Clerici and Marco O. Bertelli
}

(Information about the authors can be found at the end of this article.)

Received 3 February 2021

Revised 14 June 2021

15 July 2021

Accepted 15 July 2021

(C) Jacopo Santambrogio, Michela Russo, Sergio

Terrevazzi, Gianluca Peschi, Massimo Clerici and Marco O. Bertelli. Published by Emerald Publishing Limited. This article is published under the Creative Commons Attribution (CC BY

4.0) licence. Anyone may reproduce, distribute, translate and create derivative works of this article (for both commercial \& non-commercial purposes), subject to full attribution to the original publication and authors. The full terms of this licence may be seen at http:// creativecommons.org/licences/ by/4.0/legalcode

Sergio Cirillo, Giuseppina Redaelli, Carmela Pietrosanto, Milena Danese, Loredana Bellanova and the whole team of nurses, professional caregivers, social workers, and educators deserve special thanks for their contributions to the clinical work with study participants during the COVID-19 pandemic.

\begin{abstract}
Purpose - Persons with intellectual disability and/or low-functioning autism spectrum didorder are with high support need (ID/ASD-HSN) are among the people who are most vulnerable to the COVID-19 pandemic. The specific vulnerability and the protective factors for persons with ID/LF-ASD attending residential and rehabilitative facilities have however received little attention. This paper aims to describe how two facilities located in the Italian COVID-19 red zone faced the risks associated with the spread of the pandemic and the results they have achieved so far.
\end{abstract}

Design/methodology/approach - Interventions to contrast the spread of the pandemic and preserve clients' health conditions have been systematically monitored and recorded since the very beginning of the pandemic.

Findings - 26/138 clients had to undergo clinical screening and laboratory tests for COVID-like symptomatology, but only one resulted affected by COVID-19 and survived. Considering that Lombardy had 89,595 cases and 16,262 deaths (January-May 2020), one COVID-19 case/138 clients is a good result. Temporarily limiting physical contacts with friends/family in favor of reducing the burden of risk and adopting a system of prevention/safety strategies directed for persons with ID/LF-ASD attending and their caregivers have been useful measures.

Research limitations/implications - Structured or semi-structured interviews (using professional caregivers as informant) to confirm behavioral and emotional changes in the clients could not be carried out because of lack of time and resources (which were captured by the management of the pandemic) and could be the next goal for our residential facilities to implement the management of epidemic acute phases in a research-oriented view.

Originality/value - This study is a service evaluation report about facing COVID-19 pandemic. Only few such studies are present in medical literature about ID/ASD.

Keywords Intellectual disability, Prevention, Residential care, Autism spectrum disorder, COVID-19, Pandemic

Paper type Viewpoint

\section{Introduction}

Intellectual disability (ID) and low-functioning autism spectrum disorder (LF-ASD) represent the two neurodevelopmental disorders with the highest impact on personal functioning. They include many different clinical (syndromic) conditions (Salvador-Carulla and Bertelli, 
2008), which combine cognitive and relational impairment, with high co-occurrence rates (Baio et al., 2018; Maenner et al., 2020) and overlapping symptoms (Bertelli et al., 2020a).

Persons with ID and/or LF-ASD are among those populations most vulnerable to COVID-19 and associated sequelae, mortality and factors of mental distress (Druss, 2020; Yahya et al., 2020; Armitage and Nellums, 2020; Zhu et al., 2020; Courtenay and Perera, 2020; lasevoli et al., 2020; Turk et al., 2020).

One of the causes of such a high vulnerability is physical multi-morbidity, which includes endocrine diseases, hypertension, respiratory problems, cancer and other conditions associated with a higher risk for SARS-CoV-2 acute respiratory distress syndrome and other COVID-19 complications. Research on previous respiratory viral infections, including H1N1 and RSV, suggests that persons with genetic syndromes including ID and/or ASD (i.e. Down syndrome) are more likely than the general population to develop complications and require hospitalization. Some forms of ID/ASD have been found to present high proinflammatory cytokines as well as higher ACE expression and functionality, which have been associated with acute severe systemic inflammatory response in the pathogenesis of SARS-CoV-2 pneumonia (Theoharides and Zhang, 2011; Firouzabadi et al., 2016; De Toma and Dierssen, 2021).

Another important element contributing to people with ID/LF-ASD's sensitivity to COVID-19 and related factors of mental distress is their psychological fragility, as evidenced by a high prevalence of psychiatric disorders, with an overall lifetime prevalence up to $44 \%$ or even higher when ID and ASD co-occur. Anxiety disorders and affective disorders are the most common mental ill-health conditions. Symptoms presentation is often quite different from that of the general population, especially in persons with low or absent verbal communication skills, who may only be able to express themselves through changes in behavior. Also in terms of physical health, people with ID/LF-ASD may have difficulty conveying symptoms or even communicating when they are not feeling well. As a result, it is critical that their caregivers, both family and professional, be sensitive to the onset of observable symptoms or major changes in their behavior that might be related with a health condition so that mental and physical disorders can be identified in a timely manner (Hassiotis et al., 2020; Alexander et al., 2020).

Beside physical and mental frailty, there are several other factors that make persons with ID/ LF-ASD particularly vulnerable to COVID-19 and associated mental distress, including low levels of health literacy, low compliance with complex hygiene rules and reliance on other people for care (Buonaguro and Bertelli, 2020). They may not understand what COVID-19 pandemic is and the purpose of all the measures indicated by the national and local government as well as by the health systems to contain its spread: for example, social distancing, using masks, handwashing, avoiding touching face and eyes or sneezing into the elbow (Bertelli et al., 2020b; Courtenay and Perera, 2020).

The status of the person's living or housing accommodations (domestic, residential setting or inpatient status) has also been pointed out to impact significantly on SARS-CoV-2 spread and possible COVID-19 sequelae in persons with ID/ASD (McGonigal, 2020), with a series of advantages and disadvantages for every condition.

Congregate settings have been associated with relevant risk factors owing to the unavoidable sharing of essential living spaces and objects by a high number of persons (e.g. bedrooms, bathrooms, kitchens) (Landes et al., 2020a), but also with some protective factors such as the ability to limit and manage outside visitors, offering routine virus and symptom testing, maintaining regular surveillance of residents' health, providing prompt health interventions, guaranteeing that no person lives in isolation, working with advocacy groups to create virtual and setting-specific ways to engage with those socially isolated and working with local businesses to provide easier ways to address essential needs and services (Boyle et al., 2020; Buonaguro and Bertelli, 2020). 
The effects of COVID-19 on people with ID/LF-ASD have received little research attention, especially when it comes to different living arrangements and, more specifically, congregate settings.

In Italy, some reports have emerged on infection events with SARS-CoV-2 and severe COVID-19 symptoms and hospitalizations of persons with ID/LF-ASD in residential facilities (Ricci, 2020). The lack of official precise and well-defined guidelines addressing the emergency management at the very beginning of the pandemic has probably contributed to difficulties in caring for people in residential settings. Studies from North-East United States of America reported that the COVID-19 case rate was 4.1 times higher and case-fatality rate 1.9 times higher for people with ID living in residential group homes than the general population (Landes et al., 2020a). More recent data from California show COVID-19 outcomes for people with ID to vary by residential setting, with higher case rates in settings with more residents and higher case-fatality rates in settings that provide skilled nursing care (Landes et al., 2020b).

To our knowledge, no publication had yet provided information on the specific impact, risks, coping methods or intervention techniques associated to COVID-19 in congregate living arrangements, such as the one aimed at in this report, which pertains to two cluster centers located in Mombello - Limbiate, Italy.

\section{Methodology}

To better understand COVID-19 outcomes, protective factors and useful management strategies among people with ID and/or LF-ASD living in congregate settings, the present study used a sample of 138 adults with ID and/or ASD receiving residential and rehabilitative support in two facilities (with a capacity of 80 and 60 persons, respectively) located in Mombello - Limbiate, which is a 35,000 inhabitants town in Lombardy, the Italian area hit the hardest by COVID-19.

The area around "Mombello" has been known as an area dedicated to people suffering from mental disorders: psychiatric patients have lived for more than a century in a wide asylum (now demolished and only partially converted) of that area. In 1962, children with ID and/or ASD hosted in that asylum were moved to a new center, dedicated to the psychiatrist Giuseppe Corberi, which has been repeatedly modified over time up to the current structure that includes residential and rehabilitative facilities organized as cluster centers, with a central building for shared services and several housing units with different levels of autonomy.

The study sample includes 138 adults with ID and/or ASD, with a mean age of 58 years ( \pm 10.6) and a sex ratio (M:F) of 3:1. All clinical characteristics are summarized in Table 1.

Lombardy has been an Italian "red zone" for COVID-19 dissemination: it had 89,595 cases and 16,262 deaths (881 in Monza-Brianza area) from January to May 2020. During the same time frame in Italy, there have been 232,639 new COVID-19 cases reported, with 32,981 fatalities (ISTAT, 2020).

It was therefore very probable that such an incidence and fatality of COVID-19 spread could regard also the residential facilities for persons with ID/ASD in Monza-Brianza area.

COVID-19 diagnosis indicated that the person has been diagnosed with COVID-19 by a laboratory test during the research.

This service evaluation report has been approved by ASST-Monza Social Health Direction and the University of Milano-Bicocca. It was determined to be an "exempt research," including waiver of informed consent, as it used fully deidentified data.

\section{Findings}

Before COVID-19 outbreak, the population described in the present paper, unlike those examined in the publications listed in the introduction, resided in rehabilitation centers with 
Table 1 Clinical characteristics of the sample

Intellectual disability severity

Severe

$111(80 \%)$

Moderate

$21(15 \%)$

Mild

$6(5 \%)$

Comorbid autism spectrum disorder

$46(33 \%)$

Challenging behaviors

$32(23 \%)$

Co-occurrent psychiatric disorders

$40(29 \%)$

(at least one among: schizophrenia spectrum disorder, ADHD, mood disorder, obsessive compulsive disorder, specific phobia and personality disorder)

Severe physical illnesses

Epilepsy

Blindness and other visual impairments

Chronic obstructive pulmonary disease

Diabetes

Arnold-Chiari syndrome

Sjögren syndrome

Other

Long-term pharmacotherapy

First generation anti-psychotics

Second generation anti-psychotics

Antiepileptics

Sedatives

Antidepressants

General medical drugs

planned exits and family visits. Physical proximity to caregivers and loved ones is usually required to bridge gaps in intellectual and communication abilities and to make day-to-day life fulfilling, predictable and manageable (Constantino et al., 2020). Isolation from family members is therefore an issue for persons with ID/ASD living in residential facilities.

Since the start of the pandemic (February 2020) clinical data (vital signs and clinical conditions) for every resident in each cluster center have been registered every day in specific monitoring papers and medical record, then patients with COVID-19 suspected symptoms have been isolated and closely monitored. In those cases, nasopharyngeal swabs have been performed and the results have been put in a shared database.

Structured or semi-structured interviews (using professional caregivers as informants) to confirm behavioral and emotional changes in the clients could not be carried out because of lack of time and resources (which were focused on the management of the pandemic).

Staff members with COVID-19 suspected symptoms promptly informed the General Hospital Occupational Medicine Unit and were asked to stay at home, waiting for nasopharyngeal swabs.

Regular team meeting have been done to develop a shared safety plan inside each cluster center.

The residential facilities director was in regular contact with the General Hospital crisis unit and the local Social and Health Direction via phone or video conversations. Since the earliest phase of the pandemic (last week of February, 2020), regular team meetings have been set up to cope with the pandemic response and to create a COVID-19 safety plan, basing on the general indications provided by the Italian National Institute of Health (Rapporto ISS COVID-19 n. 2/2020 Rev.; Rapporto ISS COVID-19 n. 4/2020 Rev.), the local Health Direction (ASST-Monza, 2020) and the Italian Society for Neurodevelopmental Disorders (Bertelli et al., 2020c).

COVID-19 safety plan (summarized below) has been followed carefully by all members of the staff, both in assisting residents and in monitoring personal health conditions: 
- to comply with the safety plan, all staff were asked, before going into the facility to start their shift, to take their temperature; if it was over 37.5 degrees they were not allowed to start working;

- as the early phase staff were provided with personal protective equipment (PPE) while residents were not, because of their severe cognitive deficit and inability to understand what a mask is for;

- all the residents have been monitored every day and if their temperature was over 37.5 degrees, blood pressure, heart and respiratory rate and oxygen saturation were taken and the patient suspected of COVID-19 case was isolated in a separated specific area;

- in these specific cases, "suspected" patients were followed up by doctors and nurses, with PPE, received a first nasopharyngeal swab and a second one after two weeks;

- a screening campaign with nasopharyngeal swab and COVID-19 serology have started among the professional caregivers;

- all family members and external professional caregivers have been forbidden to enter the structures since the last week of February 2020;

- usual routine has been gradually replaced with a new routine;

- activities in the Daily Center have been re-organized with social distancing;

- promoting the understanding of what was happening and what needed to be done with mild ID clients; and

- staying connected with key contacts (friends, family members, etc.) through video calls.

PPE, including gloves, gowns and eye and mouth protection (both surgical mask and FFP2 mask, depending on the level of assistance), have been available every day for each member of the staff since the beginning of the pandemic and throughout its progress.

Of the 138 residents living in the two services, only 26 needed to be tested for COVID-19 and only one was found to be positive at nasopharyngeal swab at the end of March 2020. At the end of April 2020 however he was back to negative and healthy.

A total of 98 of the 256 professional caregivers were tested for COVID-19 using a nasopharyngeal swab, and six were positive, but all of them became negative after 10 days without displaying any relevant symptoms. COVID-19 serology was used to screen all 256 professional carers, and 11 of them tested positive, with one of them also testing positive on a nasopharyngeal swab. Exits and external activities for cluster center residents were restricted, while inside activities were modified to social distance and other safety rules. As an example, for individuals who used to go to the outside cafeteria on a daily basis to meet people and have some fun, a meeting area was set up within the clusters, and beverages were delivered by professional caretakers at the usual break-time. Self-expression through visual art and music was strongly encouraged and favored. Clients who inquired as to why staff members wore masks and what the pandemic was, as well as their grief over the distance between them and their parents, were given more particular support.

Since the epidemic began, all residents have had access to a well-equipped internet service, allowing them to conduct frequent video conversations with family members and other significant persons.

\section{Discussion and conclusions}

This study was a naturalistic observational study of a pandemic event (COVID-19 impact on residential facilities for persons with ID and/or LF-ASD), so its methodology could not be planned in advance. Data are limited to what was collected during the pandemic. 
In the two residential and rehabilitative facilities studied, only one client out of 138 was infected with COVID-19 $(0.7 \%)$, which may be considered a significant outcome. The only data available in the literature that can be compared to ours are those provided by Mills and colleagues (2020), however there are significant differences. Mills and colleagues report how their organization in the 100-day period between 20 January 2020 and 30 April 2020 provided continuous support for 11,540 individuals with IDD. In total $64 \%$ of individuals were in residential, community settings, and $36 \%$ were in intermediate care facilities. A total of 122 individuals with IDD were placed in quarantine for showing symptoms and signs of acute infection such as fever or cough. A total of 66 individuals tested positive for SARS-CoV-2. A total of 15 COVID-19-positive individuals were hospitalized. As of 30 April 2020, seven of the individuals hospitalized had been discharged, sent back home and were in the process of recovering. Five remained hospitalized, with three improving and two remaining in intensive care and on mechanical ventilation. There have been three deaths (Mills et al., 2020).

Overall, we observed that a well-coordinated and well-communicated infection management, personnel screening and visitor disruption helped to keep COVID-19 to a minimum in our congregate settings, which is aligned with findings reported by Mills and collaborators (Mills et al., 2020). Despite the fact that we were aware that staff members may be infection carriers, we could not rationally decrease or modify their shifts owing to the necessity to provide full clinical support to people with ID/LF-ASD. Many parents suffered the prolonged impossibility to come and visit their loved ones, but this was somewhat alleviated by the development of an effective video-call service, with the help of family associations and our social workers' team. Some family members wondered whether their sibling with disability was able to recognize them through the video call device, as the body was not involved anymore and replaced by a small image. Another major concern addressed the extent to which their siblings understood the level of severity of COVID-19 pandemic and complied with safety measures. We did our best to reassure all of them by providing detailed descriptions and pictures of the skills acquired by their siblings. We could also reassure them that the lack of physical contact with family members did not cause unmanageable psychological and behavioral alterations.

On 3 August 2020, after five months, family members were allowed back to visit their siblings, but only with short scheduled appointments and following social distancing and other safety rules. Some persons with disability tried to embrace their family members or expected to receive food or objects from their hands but staff promptly prevented close contacts and did their best to manage consequent disappointment.

Considering that Lombardy had 89,595 cases and 16,262 deaths (5,500 cases and 881 deaths in Monza-Brianza area, 878,267 inhabitants) from January to May 2020, we could assume that one COVID-19 case out of the 138 clients of the two residential facilities, and no deaths, is a good result.

Limiting physical contact with family and friends to reduce the risk of infection, as well as implementing a system of safety measures for people with ID/LF-ASD and their carers, have proven to be beneficial (Buonaguro and Bertelli, 2020; Bertelli et al., 2020c).

This compromise to the individuals' freedoms that occurred as a result of some of the measures that were taken to prevent the spread of COVID-19 could have caused distress in the clients, but professional caregivers who daily observed them, have not reported behavioral changes, nor other signs of significant distress, nor worsening of previous comorbid psychiatric conditions. This may have also happened as a result of the multiprofessional team's involvement in terms of communication and psychological support. Because of a lack of time and resources (catalyzed on pandemic management), structured or semi-structured interviews to confirm behavioral and emotional changes in clients could not be conducted. This could be the next goal for our residential facilities to implement pandemic acute phase management in a research-oriented manner. Our findings are in 
contrast with those of other studies indicating a significant association between number of residents living in a setting and COVID-19 diagnosis (Landes et al., 2020b, 2021). Based upon our result, it is to reconsider whether the number of residents alone is an actual risk for more severe outcomes, or possibly a proxy for other factors. In a recent study including 543 individuals with ID, Landes and collaborators found the COVID-19 diagnosis to be related to the type of residential service only in univariable analysis, and not in multivariable analysis (Landes et al., 2021).

\section{Author contributions}

Conceptualization, M.O.B., J.S., M.R., S.T., M.C.; methodology, M.O.B., J.S., S.T.; validation, M.O.B.; formal analysis, J.S.; investigation, J.S., M.R., S.T; resources, all authors; data curation, J.S., S.T, M.R.; writing - original draft preparation, J.S. and M.O.B.; writing - review and editing, M.O.B. and J.S.; supervision, M.O.B and M.C.; project administration, J.S., M.O.B., M.C., G.P.; funding acquisition, S.T, M.C., G.P. All authors have read and agreed to the published version of the manuscript.

\section{References}

Alexander, R., Ravi, A., Barclay, H., Sawhney, I., Chester, V., Malcolm, V., Brolly, K., Mukherji, K., Zia, A., Tharian, R., Howell, A., Lane, T., Cooper, V. and Langdon, P.E. (2020), "Guidance for the treatment and management of COVID-19 among people with intellectual disabilities", Journal of Policy and Practice in Intellectual Disabilities, Vol. 17 No. 3.

Armitage, R. and Nellums, L.B. (2020), "The COVID-19 response must be disability inclusive", The Lancet Public Health, Vol. 5 No. 5, p. e257.

ASST-Monza (2020), "Coronavirus COVID-19 indicazioni igienico sanitarie — versione del 24 marzo 2020 - 17 aprile 2020", Azienda Socio Sanitaria Territoriale.

Baio, J., Wiggins, L., Christensen, D.L., Maenner, M.J., Daniels, J., Warren, Z., Kurzius-Spencer, M., Zahorodny, W., Robinson Rosenberg, C., White, T., Durkin, M.S., Imm, P., Nikolaou, L., Yeargin-Allsopp, M., Lee, L.C., Harrington, R., Lopez, M., Fitzgerald, R.T., Hewitt, A., Pettygrove, S., Constantino, J.N., Vehorn, A., Shenouda, J., Hall-Lande, J., VAN Naarden Braun, K. and Dowling, N.F. (2018), "Prevalence of autism spectrum disorder among children aged 8 years - autism and developmental disabilities monitoring network, 11 sites, United States, 2014", MMWRSurveill Summ, Vol. 67, pp. 1-23.

Bertelli, M.O., Salvador-Carulla, L., Munir, K.M., Scattoni, M.L., Azeem, M.W. and Javed, A. (2020a), "Intellectual developmental disorder and autism spectrum disorder in the WPA next triennium mainstream", World Psychiatry, Vol. 19 No. 2, p. 260.

Bertelli, M.O., Buonaguro, E.F., Fiengo, A.L.C., Artoni, V., Zorzi, S., Scuticchio, D., Cappa, C., Armellini, M., Panocchia, N., Lo Presti, E., Ghelma, F., Keller, R., Corti, S., Rossi, M., Bianco, A., Di Sarro, R., Varrucciu, N. and Scattoni, M.L. (2020c), "Consigli per gli operatori sanitari coinvolti nella cura e nell'assistenza alla persona con disabilità intellettiva e autismo con necessità elevata e molto elevata di supporto, v.1.3", available at: www.sidin.org/2020/05/scudo-psicologico-pergli-operatori-sanitari/

Bertelli, M.O., Scuticchio, D., Bianco, A., Buonaguro, E.F., Laghi, F., Ghelma, F., Rossi, M., Vannucchi, G., Cavagnola, R., Chiodelli, G., Corti, S., Leoni, M., Gusso, S., Cappa, C., Filighera, L., Simone, S., Zunino, M., Belotti, R., Perrone, F., Di Sarro, R., Keller, R., Arduino, M., Brondino, N., Politi, P., M, Lupi, M, B., Lo Presti, E., Pace, P., Armellini, M., Santambrogio, J., Barbui, C., Papola, D., Ceccarani, P., Franchini, R., Bergamin, C. and Scattoni, M.L. (2020b), "Advice for managing the COVID-19 outbreak and the associated factors of mental distress for people with intellectual disability and autism spectrum disorder with high and very high support needs", available at: https://3ba346de-fde6-473f-b1da536498661f9c. filesusr.com/ugd/e172ł3_3a189161289644fb9c5eb993c8192e7f.pdf

Boyle, C.A., Fox, M.H., Havercamp, S.M. and Zubler, J. (2020), "The public health response to the COVID-19 pandemic for people with disabilities", Disability and Health Journal, Vol. 13 No. 3, p. 100943.

Buonaguro, E.F. and Bertelli, M.O. (2020), "COVID-19 and intellectual disability/autism spectrum disorder with high and very high support needs: issues of physical and mental vulnerability", Advances in Mental Health and Intellectual Disabilities, Vol. 15 No. 1, pp. 8-19. 
Constantino, J.N., Sahin, M., Piven, J., Rodgers, R. and Tschida, J. (2020), "The impact of COVID-19 on individuals with intellectual and developmental disabilities: clinical and scientific priorities", American Journal of Psychiatry, Vol. 177 No. 11

Courtenay, K. and Perera, B. (2020), "COVID-19 and people with intellectual disability: impacts of a pandemic", Irish Journal of Psychological Medicine, Vol. 37 No. 3.

De Toma, I. and Dierssen, M. (2021), "Network analysis of down syndrome and SARS-CoV-2 identifies risk and protective factors for COVID-19", Scientific Reports, Vol. 11 No. 1, p. 1930.

Druss, B.G. (2020), "Addressing the COVID-19 pandemic in populations with serious mental illness", JAMA Psychiatry, Vol. 77 No. 9.

Firouzabadi, N., Ghazanfari, N., Alavi Shoushtari, A., Erfani, N., Fathi, F., Bazrafkan, M. and Bahramali, E. (2016), "Genetic variants of Angiotensin-Converting enzyme are linked to autism: a Case-Control study", PLoS One, Vol. 11 No. 4,p. e0153667.

Hassiotis, A., Ali, A., Courtemanche, A., Lunsky, Y., Lee Mclntyre, L., Napolitamo, D., van der Nagel, J. and Werner, S. (2020), "In the time of the pandemic: safeguarding people with developmental disabilities against the impact of coronavirus", Journal of Mental Health Research in Intellectual Disabilities, Vol. 13 No. 2, pp. 63-65.

lasevoli, F., Fornaro, M., D'Urso, G., Galletta, D., Casella, C., Paternoster, M., Buccelli, C., De Bartolomeis, A. and Group, C.-I.P.S. (2020), "Psychological distress in patients with serious mental illness during the COVID19 outbreak and one-month mass quarantine in Italy", Psychological Medicine, Vol. 51 No. 6, pp. 1-3.

ISTAT (2020), "Impatto dell'epidemia COVID-19 sulla mortalità totale della popolazione residente periodo gennaio-maggio 2020", available at: www.istat.it/it/files/2020/07/Rapp_Istat_Iss_9luglio.pdf

Landes, S.D., Turk, M.A. and Wong, A.W.W.A. (2020b), "COVID-19 outcomes among people with intellectual and developmental disability in California: the importance of type of residence and skilled nursing care needs", Disability and Health Journal, Vol. 14 No. 2, p. 101051.

Landes, S.D., Turk, M.A., Damiani, M.R., Proctor, P. and Baier, S. (2021), "Risk factors associated with COVID-19 outcomes among people with intellectual and developmental disabilities receiving residential services", JAMA Network Open, Vol. 4 No. 6, p. e2112862.

Landes, S.D., Turk, M.A., Formica, M.K., McDonald, K.E. and Stevens, J.D. (2020a), "COVID-19 outcomes among people with intellectual and developmental disability living in residential group homes in New York state", Disability and Health Journal, Vol. 13 No. 4, p. 100969.

McGonigal, M. (2020), "Providing quality care to the intellectually disadvantaged patient population during the COVID-19 pandemic", Critical Care Nursing Quarterly, Vol. 43 No. 4, pp. 480-483.

Maenner, M.J., Shaw, K.A., Baio, J.E.D.S., Washington, A., Patrick, M., Dirienzo, M., Christensen, D.L., Wiggins, L.D., Pettygrove, S., Andrews, J.G., Lopez, M., Hudson, A., Baroud, T., Schwenk, Y., White, T., Rosenberg, C.R.L.E.E.L.C., Harrington, R.A., Huston, M., Hewitt, A.P.H.D., Esler, A., Hall-Lande, J., Poynter, J. N., Hallas-Muchow, L., Constantino, J.N., Fitzgerald, R.T., Zahorodny, W., Shenouda, J., Daniels, J.L., Warren, Z., Vehorn, A., Salinas, A., Durkin, M.S. and Dietz, P.M. (2020), "Prevalence of autism spectrum disorder among children aged 8 years - autism and developmental disabilities monitoring network, 11 sites, United States, 2016", Mmwr. Surveillance Summaries, Vol. 69 No. 4, pp. 1-12.

Mills, W.R., Sender, S., Lichtefeld, J., Romano, N., Reynolds, K., Price, M., Phipps, J., White, L., Howard, S., Poltavski, D. and Barnes, R. (2020), "Supporting individuals with intellectual and developmental disability during the first 100 days of the COVID-19 outbreak in the USA", Journal of Intellectual Disability Research, Vol. 64 No. 7, pp. 489-496.

Ricci, G. (2020), Coronavirus, i Centri per Disabili Sono il Fronte Dimenticato Dell'emergenza, Corriere della Sera.

Salvador-Carulla, L. and Bertelli, M. (2008), "Mental retardation or intellectual disability: time for a conceptual change", Psychopathology, Vol. 41 No. 1, pp. 10-167.

Theoharides, T.C. and Zhang, B. (2011), "Neuro-inflammation, blood-brain barrier, seizures and autism", Journal of Neuroinflammation, Vol. 8 No. 1, p. 168.

Turk, M.A., Landes, S.D., Formica, M.K. and Goss, K.D. (2020), "Intellectual and developmental disability and COVID-19 case-fatality trends: triNetX analysis", Disability and Health Journal, Vol. 13 No. 3, p. 100942.

Yahya, A.S., Khawaja, S. and Chukwuma, J. (2020), "The impact of COVID-19 in psychiatry", Prim Care Companion CNS Disord, Vol. 22. 
Zhu, Y., Chen, L., Ji, H., Xi, M., Fang, Y. and Li, Y. (2020), "The risk and prevention of novel coronavirus pneumonia infections among inpatients in psychiatric hospitals", Neuroscience Bulletin, Vol. 36 No. 3, pp. 299-302.

\section{Further reading}

Istituto Superiore Sanità, Rapporto ISS COVID-19 n. 4/2020 Rev "Indicazioni ad interim per la prevenzione e il controllo dell'infezione da SARS-CoV-2 in strutture residenziali sociosanitarie e socioassistenziali",

Istituto Superiore Sanità, Rapporto ISS COVID-19 n. 2/2020 Rev "Indicazioni ad interim per un utilizzo razionale delle protezioni per infezione da SARS-COV-2 nelle attività sanitarie e sociosanitarie (assistenza a soggetti affetti da COVID-19) nell'attuale scenario emergenziale SARS-CoV-2",

\section{Author affiliations}

Jacopo Santambrogio is based at the Department of Medicine and Surgery, University of Milano-Bicocca, Milan, Italy; Presidio Corberi, ASST Brianza, Limbiate, Italy and Adele Bonolis AS.FRA. Foundation, Vedano al Lambro, Italy.

Michela Russo and Sergio Terrevazzi are based at the RSD Beato Papa Giovanni XXIII and Presidio Corberi, ASST Brianza, Limbiate, Italy.

Gianluca Peschi is based at the Social and Health Direction, ASST di Monza, Monza, Italy.

Massimo Clerici is based at the Department of Medicine and Surgery, University of MilanoBicocca, Milan, Italy and Department of Mental Health and Addiction, ASST di Monza, Monza, Italy.

Marco O. Bertelli is based at the Research and Clinical Center (CREA), San Sebastiano Foundation, Misericordia di Firenze, Florence, Italy.

\section{Corresponding author}

Jacopo Santambrogio can be contacted at: j.santambrogio@gmail.com

For instructions on how to order reprints of this article, please visit our website: www.emeraldgrouppublishing.com/licensing/reprints.htm

Or contact us for further details: permissions@emeraldinsight.com 